\title{
The role of levetiracetam in treatment of seizures in brain tumor patients
}

\author{
Ekokobe Fonkem ${ }^{1,2}$, Paul Bricker ${ }^{2}$, Diana Mungall ${ }^{2}$, Jose Aceves ${ }^{2,3}$, Eromata Ebwe ${ }^{1}$, Wei Tang ${ }^{1,2}$ and \\ Batool Kirmani ${ }^{2,3}$
}

${ }^{1}$ The Brain Tumor Center, Scott \& White Healthcare, Temple, TX, USA

${ }^{2}$ Texas A\&M Health Science Center College of Medicine, Temple, TX, USA

${ }^{3}$ Epilepsy Center, Scott and White Neuroscience Institute, Temple, TX, USA

\section{Edited by:}

Jorge Asconape, Loyola University, USA

\section{Reviewed by:}

Peter Halasz, Hungarian Sleep

Society, Hungary

Abhay Sharma, Council of Scientific

and Industrial Research, India

\section{*Correspondence:}

Ekokobe Fonkem, Scott \& White

Healthcare, 2401 South 31st Street,

Temple, TX 76504, USA

e-mail:efonkem@sw.org
Levetiracetam, trade name Keppra, is a new second generation antiepileptic drug that is being increasingly used in brain tumor patients. In patients suffering with brain tumors, seizures are one of the leading neurologic complications being seen in more than $30 \%$ of patients. Unlike other antiepileptic drugs, levetiracetam is proposed to bind to a synaptic vesicle protein inhibiting calcium release. Brain tumor patients are frequently on chemotherapy or other drugs that induce cytochrome P450, causing significant drug interactions. However, levetiracetam does not induce the P450 system and does not exhibit any relevant drug interactions. Intravenous delivery is as bioavailable as the oral medication allowing it to be used in emergency situations. Levetiracetam is an attractive option for brain tumor patients suffering from seizures, but also can be used prophylactically in patients with brain tumors, or patients undergoing neurological surgery. Emerging studies have also demonstrated that levetiracetam can increase the sensitivity of Glioblastoma tumors to the chemotherapy drug temozolomide. Levetiracetam is a safe alternative to conventional antiepileptic drugs and an emerging tool for brain tumor patients combating seizures.

Keywords: intravenous levetiracetam, seizures, brain tumor patients, antiepileptic drugs, neurologic complications

\section{INTRODUCTION}

Individuals with brain tumors represent a very challenging patient population for clinicians. Not only must clinicians deal with and treat the primary tumor, but they must also manage the numerous accompanying sequelae. Seizures are commonly seen in brain tumor patients, with reports of $30 \%$ or more depending on the type of tumor (1). In fact, an epileptic seizure is the presenting symptom of a tumor in $30-50 \%$ of patients, and $10-30 \%$ of those patients will go on to develop recurrent seizures over the course of the disease $(2,3)$. The presence of seizures and convulsions has been shown to add substantial morbidity to these patients (4).

\section{ROLE OF ANTICONVULSANTS IN ACUTE SEIZURE MANAGEMENT AND SEIZURE PROPHYLAXIS IN BRAIN TUMOR PATIENTS}

Current consensus states that all patients with brain tumors should be treated with antiepileptic drugs. Currently, the first-line medications including phenytoin, carbamazepine, or valproate all have demonstrated major side-effects and dangerous drug interactions with commonly used chemotherapy and tumor medications. The most common side-effects seen with these highly used antiepileptic medications include cognitive impairment, bone-marrow suppression, liver dysfunction, and dermatological symptoms (5). Reinforcing the complexity of care in these cases are several studies demonstrating that side-effects are more frequent in patients with brain tumors compared with the overall epileptic population $(3,6)$. Tsai et al. conducted a retrospective chart review to assess the effect of valproic acid (VPA) on the outcome of patients with glioblastoma multiforme (GBM). The electronic medical records were queried from January 2004 to December 2006 and 102 patients newly diagnosed with GBM were found. Those patients were followed until January 2010 and over that time 87 patients died due to disease progression, 7 patients lived and were followed between 43.4 and 61.0 months, and 8 patients were lost to follow up. VPA was administered at a starting dose of $400 \mathrm{mg}$ every $8 \mathrm{~h}$ in the form of a sodium salt. It was then adjusted as needed to serum level or seizure activity. Patients were analyzed in two groups: an "early" treatment group in which the patients began VPA treatment within 2 weeks of initial diagnosis and the "late" treatment group where the patients began treatment with VPA more than 2 weeks after initial diagnosis.

Seven patients on VPA therapy provided tissue samples from a second resection procedure for analysis of histone acetylation. When these samples were analyzed, there was an increase in acetylation in a small subset of patients. Eighty-five of 102 patients $(83.3 \%)$ underwent radiotherapy, $61 / 102$ patients $(59.8 \%)$ underwent chemotherapy, and all patients had a neurosurgical procedure with the most common being total excision (47.1\%). Thirtythree of 102 patients had VPA therapy and of those 16 patients (58.5 serum concentration) started therapy less than a week after diagnosis and 17 patients (52.4 serum concentration). The early treatment group had an average serum VPA level of $58.46 \mu \mathrm{g} / \mathrm{ml}$ (34.1-72.6) and the late group was $52.37 \mu \mathrm{g} / \mathrm{ml}$ (36.3-73.2). When the early treatment group was evaluated with univariate analysis there appeared to be a survival benefit conferred $(P=0.035)$. However, when a stratified analysis according to chemotherapy 
was used, VPA therapy was not associated with a statistically significant difference $(P=0.315)$ in overall survival. Mild nausea and thrombocytopenia were the most commonly reported side effect (11/33 and 11/33, respectively). The authors concluded that VPA therapy did not affect patient survival significantly (7).

Drug interactions between antiepileptic drugs and commonly used tumor therapies can lead to inadequate control of the seizures or sub-therapeutic treatment of the tumor. Toxic effects have also been noted, leading to unnecessary morbidity. Many of the common antiepileptic drugs induce cytochrome P450 enzymes which cause faster metabolism and lower concentrations within plasma (5). Corticosteroids and many other chemotherapy drugs exhibit decreased effectiveness in the presence of enzyme activating antiepileptic drugs $(8,9)$. The reciprocal relationship is also seen as there are chemotherapeutic agents that induce enzymes of the P450 system, lowering concentrations of antiepileptic drugs $(3,10)$.

Within the past 10 years, several new antiepileptic drugs including lamotrigine, levetiracetam, oxcarbazepine, lacosamide, topiramate, and zonisamide have emerged that are without clinically relevant drug interactions (11-13). Data are limited for these newer agents and the main limitation in use is lack of intravenous formulation. Maschio et al. studied the effects of 12 months of oxcarbazepine (OXC) monotherapy on seizure control in patients with brain tumor-related epilepsy (BTRE) in a prospective, observational study. Eleven women and 14 men (mean age 49.7 years, range 25-75) with BTRE were enrolled between September 2007 and January 2009. Enrolled subjects had a histological diagnosis of meningiomas, primary grade I gliomas, low grade gliomas, anaplastic gliomas, or multiform glioblastoma. Epileptic patients were eligible if they had simple or complex seizures with or without secondary generalization, if they had greater than or equal to two seizures per month on no AEDs before referral to their center, or patients that had been treated at the maximum tolerated dosage with other AEDs. Twenty-four patients received monotherapy with OXC of which 17 patients were de novo and 7 patients rare on monotherapy with one AED. A baseline was established at the first visit of seizure frequency, neurological examination, Zung self-depression rating scale (ZSDRS), adverse events profile, and patients were given a seizure diary. During week one OXC began and any prior AED was tapered gradually over the first 3 weeks. The OXC dose started at $300 \mathrm{mg} /$ day and was titrated every 4 days by $300 \mathrm{mg} /$ day up to $2,100 \mathrm{mg} /$ day over 4 weeks.

The mean follow up duration was 7.1 months (range 112 months). Five patients died as a result of tumor progression and 10 patients dropped out due to severe side-effects $(N=6)$, uncontrolled seizures $(N=3)$, and a lung complication $(N=1)$. Six patients $(24 \%)$ had severe side-effects due to rash $(N=4)$, confusion $(N=1)$, and dizziness $(N=1)$ and one patient $(4 \%)$ had a mild rash. Four patients had no systemic therapy, 1 had radiotherapy, 4 had chemotherapy, and 16 had both radiotherapy and chemotherapy. Patients received a mean OXC dosage of $1,230 \mathrm{mg} /$ day (range $600-2,100 \mathrm{mg} /$ day). Of the 10 patients who completed the 12 months of follow up, there was a significant seizure reduction $(P=0.005)$ in the mean weekly seizure number from $2.62 \pm 6.35$ at baseline to $0.13 \pm 0.37$ in the final follow up. There was a significant difference in the seizure freedom rate $(P=0.002)$ by the McNemar's test between the baseline and final follow up intent to treat population. Using logistic regression analysis, the authors found that the efficacy of OXC in seizure control was not affected by chemotherapy and radiotherapy $(P=0.658)$. The ZSDRS showed significantly increased mood in patients in the final follow up $(P=0.011)$. Thus, the researchers concluded that OXC was efficacious in controlling seizures. However, lack of intravenous formulation limits its use in acute cases. The other agent used is lamotrigine which is also not available in intravenous formulation (14).

Meyer et al. conducted a prospective study in order to determine the protein binding and distribution of LTG in serum, brain tissue, and brain tumor in three female and eight male subjects with brain tumors. From 1994 to 1996, 11 patients had neurosurgical operations for benign tumors $(N=2)$ and malignant tumors $(N=9)$. The subjects were aged 33-68 years (mean 56 years). Patients were enrolled if in the days immediately preceding neurosurgery they showed signs of seizures or if their preoperative EEG showed symptoms of epilepsy. For intraoperative and postoperative seizure control, subjects were administered LTG with PHT in seven patients or LTG with PHT and CBZ in three patients. Patients received a mean of 54.4 days of preoperative treatment (range 1 day-17 months) with LTG, and $2 \mathrm{~h}$ preoperatively were given a dose of $100-200 \mathrm{mg} /$ day in addition to PHT or PHT and CBZ. After administration, serial blood samples were taken from 0.5 to $12.5 \mathrm{~h}$, as well as an intraoperative blood sample and intraoperative removed tumor tissue $(N=6)$. HPLC was used to assay LTG concentrations and an ultrafiltration system was used to determine plasma binding of LTG.

The LTG concentration at the time of tumor sectioning was on average $3.7 \mu \mathrm{g} / \mathrm{ml}$ (range 1.1-9.8) in the serum, an average of $6.8 \mu \mathrm{g} / \mathrm{g}$ (range 1.0-14.9) in the brain tissue, and an average of $4.4 \mu \mathrm{g} / \mathrm{g}$ (range 2.0-8.3) in the tumor tissue. The resultant brain/serum ratio was 2.8 and the tumor/serum ratio was 1.9. The protein binding of LTG was determined to be a lipophile AED. The researchers concluded that LTG penetrates well into brain and tumor tissue and had a moderately high protein binding (15). It has been shown that pregabalin has been used as adjunctive therapy in this subgroup of patients although limited data are available, which is the case with most other newer agents (16).

Lacosamide is one of the newest agents which has the advantage of being available in both an oral and intravenous formulation. Few studies reported the efficacy as an adjunctive agent, and data are limited at this time.

Saria et al. conducted a retrospective chart review to study the use of lacosamide in patients with brain tumors. Seventy patients with primary brain tumors who received lacosamide for seizure control were identified by reviewing the medical records of five United States medical centers with brain tumor programs. Primary tumors included glioblastoma (40\%), grade II gliomas (36\%), and were followed by grade III anaplastic astrocytomas, anaplastic/atypical meningiomas, anaplastic ependymomas, and pleomorphic xanthoastrocytomas. Seventy-eight percent of patients $(N=55)$ had partial seizures and $17 \%$ had of patients $(N=12)$ had generalized seizures. Subjects had a mean age of 51 years of age. Eighty-four percent of patients had chemotherapy $(N=59)$ and $81 \%$ had radiation therapy $(N=57)$. The majority of patients 
had a surgical procedure performed, including $63 \%$ had a craniotomy, $23 \%$ had a biopsy, and $14 \%$ had both. Most patients (83\%) were on an additional AED as well as lacosamide, which was most commonly levetiracetam. The cause for the addition of lacosamide therapy was most commonly recurrent seizures (74\%) or toxicity from another AED (23\%).

A decrease in seizure frequency was found in 46 patients $(66 \%)$ and seizure control remained unchanged in 21 patients $(30 \%)$. A greater than $50 \%$ decrease in seizure frequency was achieved in 38 patients $(83 \%)$. No medication toxicities were reported in $77 \%$ of patients $(N=54)$ receiving lacosamide. The most common toxicity reported was fatigue noted by four subjects $(6 \%)$. The researchers concluded that in patients with brain tumors lacosamide was well tolerated and an active add-on AED (17).

Maschio et al. also conducted a case series of 14 patients with BTRE that had not obtained adequate seizure control on other AED treatment. Patients were consecutively recruited if they had at least one seizure per month prior to study recruitment. Lacosamide was the first-fifth add-on AED therapy and was started at $100 \mathrm{mg} /$ day and titrated weekly by $100 \mathrm{mg} /$ day to a maximum dosage of $400 \mathrm{mg} /$ day.

Follow up occurred in subjects at a mean of 5.4 months (range $<1-10$ months). During this period, nine patients died from tumor progression, no patients' underwent radiation therapy, and nine patients underwent chemotherapy. The mean lacosamide dosage was $332.1 \mathrm{mg} /$ day (range $100-400 \mathrm{mg} /$ day). One subject dropped out due to dizziness and blurred vision. The mean seizure frequency at baseline was 15.4 per month and at follow up decreased to 1.9 per month. Subjects had a median seizure reduction percentage of $79.8 \%$. Five patients $(35.7 \%)$ had a greater than $50 \%$ seizure reduction and six patients $(42.9 \%)$ were seizure free. One patient $(7.1 \%)$ had an unmodified seizure frequency. There was a statistically significant difference in the mean monthly seizure frequency from baseline to follow up $(P=0.022)$. The authors concluded that lacosamide is a valid alternative add-on AED in patients with BTRE (18) have had seizures.

\section{ROLE OF LEVETIRACETAM IN ACUTE SEIZURE MANAGEMENT AND SEIZURE PROPHYLAXIS IN BRAIN TUMOR PATIENTS}

In this article we chose to focus on the use of levetiracetam. In our experience we have witnessed better efficacy and there seems to be more evidence within the literature supporting levetiracetam in terms of seizure management. An important point to make here is that there are many antiepileptic drug choices, but very little comparative effectiveness data to help physicians decide the best AED to use in the innumerably variable clinical scenarios. This paper attempts to make a contribution and demonstrate what we feel is a good drug choice for managing seizures in the unique population of patients with brain tumors. Levetiracetam is very different from the more commonly used antiepileptic drugs $(19,20)$. Its action is believed to involve neuronal binding to synaptic vesicle protein 2A (SV2A). Binding to this protein somehow acts as an inhibitor of synaptic vesicle exocytosis (21) decreasing presynaptic neurotransmitter release (21). The mechanisms of action for the more commonly used antiepileptic drugs including benzodiazepines and barbiturates affect gamma-aminobutyric acid potentiation, calcium channels, or sodium channels (22).

Levetiracetam exhibits a relative bioavailability of $100 \%$ following both oral (23) and intravenous administration. Levetiracetam, given intravenously, is considered bioequivalent to oral tablets and is well tolerated (12). Being able to give this drug intravenously is an extremely attractive trait which makes treatment in emergency or perioperative situations a possibility. Perhaps more importantly is the fact that levetiracetam is not extensively metabolized by the cytochrome P450 system (23). This is in extreme contrast to the most commonly used antiepileptic drugs. Levetiracetam has not been shown to cause any induction or inhibition of the other important cytochrome P450 enzymes including uridine diphosphate-glucuronyl-transferase or epoxide hydroxylase (23). Levetiracetam has repeatedly been shown to exhibit a low potential for clinically relevant pharmacokinetics both with other antiepileptic drugs or drugs that could possibly be used to treat brain tumors $(13,23)$. Studies have demonstrated no or very few side-effects with levetiracetam treatment in patients with brain tumors who also received antineoplastic agents $(7,24-26)$. A few of the larger studies were able to report the occasional occurrence of somnolence with initial doses of levetiracetam (27).

\section{SEIZURE MANAGEMENT WITH LEVETIRACETAM}

Over the past 10 years, there have been several studies that looked at both the effectiveness and safety of using levetiracetam in brain tumor patients. Levetiracetam, as both an adjunct therapy and a monotherapy, has shown a complete seizure control rate of between 47.4 and $100 \%$ (7,25-27). Reductions in seizures by more than $50 \%$ were recorded in a majority of articles within the literature. These numbers were extremely variable ranging from 29 to $100 \%$. However most of these studies were conducted with limited numbers of patients and were mostly retrospective analyses $(6,11,24,25,28-32)$. The two largest studies and the ones most likely indicative of what clinicians will see in their patients are from a 2005 Neurology supplement by Stevens et al. (31) and a report from Rosati et al. (27) in 2010. The study in Neurology reviewed the medical charts of 278 patients with varying brain tumors treated with levetiracetam over a 36-month period. They witnessed a greater than $50 \%$ reduction in seizure activity in over $60 \%$ of their patients. The second largest study, by Rosati et al., involved 176 patients in a prospective study over a 3-year period (27). In this study, $91 \%$ of patients were seizure free with a monotherapy of levetiracetam. Forty-nine of the patients $(60 \%)$ experienced fast and long-lasting seizure control with initial doses of $1,500-3,000 \mathrm{mg} /$ day. In 23 patients $(31.5 \%)$ an increase in the dosage up to $3,000-4,000 \mathrm{mg} /$ day was necessary because of subtherapeutic drug levels. The authors experienced no relevant laboratory abnormalities (27). Levetiracetam has demonstrated good potential however; larger cohorts over more extended periods of time would be useful.

While levetiracetam has demonstrated good potential as an adjunct therapy as well as monotherapy, several alternative uses for levetiracetam are currently being explored such as refractory status epilepticus.

Traditional seizure medications have proven woefully ineffective with over half of brain tumor patients continuing to have 
seizures despite treatment (32). Status epilepticus is seen in up to $26 \%$ of these cases (6) with the overall mortality rate reaching 30-40\% (33). First-line treatment for status epilepticus in this patient population is a combination of benzodiazepines and phenytoin (34), which has an efficacy rate of 60-70\%. Refractory status epilepticus requires an additional anesthetic drug such as propofol or midazolam to induce an iatrogenic coma (35). Recent data suggest that an alternative combination of phenytoin and levetiracetam has proven to be a very effective cocktail that does not subject the patient to mechanical ventilation or sedation (35).

Swisher et al. conducted a retrospective chart review of electronic medical records for all patients with a diagnosis of primary or metastatic brain tumor who presented with complex partial refractory status epilepticus and received Trifecta (intravenous PHT, LEV, and oral PGB). All patients were $>18$ years of age and presented between January 2006 and December 2009. Study subjects ages ranged from 25 to 84 with an average age of 56.9. There was a prior history of seizures in $70 \%$ of patients. GBM occurred in $52 \%$ of patients and was the most common tumor type, yet the tumor locations of all subjects tumors was highly variable. Ninety-one percent of patients had underwent resection for their brain tumor. Thirty-nine percent of patients received radiation, $39 \%$ received chemotherapy, and within 1 month of RSE onset $52 \%$ underwent a biopsy or brain tumor resection. PHT or LEV was used as first-line therapy in all patients and pregabalin was typically used as second or third-line therapy. The median dosage of LEV was $3,000 \mathrm{mg} /$ day and the median dosage of PGB was $375 \mathrm{mg} /$ day.

Ninety-one percent of patients were already on one AED when RSE was diagnosed, and it was statistically significant $(P=0.03)$ that more patients in the responder group were on an AED at baseline (100 vs. 71\%). The average PHT blood level in the Trifecta responder patients was 18.9 at the time of SE cessation. After the administration of Trifecta $30 \%$ of patients' (7/23) seizure frequencies were unchanged. After the administration of Trifecta, status epilepticus ceased in $70 \%$ of patients (16/23). On average, $24 \mathrm{~h}$ after the addition of the third AED status epilepticus was aborted. There was a zero mortality rate in the responder group and only one patient in the responder group required intubation. There were no adverse reactions to Trifecta reported. The authors concluded that in patients with brain tumors presenting in RSE that Trifecta use is highly effective and safe (16).

\section{SURGICAL PROPHYLAXIS}

Patients with brain tumors often must undergo neurological surgery for resection or biopsy, which in and of itself can increase risk of seizures. So controlling seizures in the perioperative phase of brain tumor management is an important consideration $(3,36)$.

Bahr et al. performed an open-label, prospective, single-arm study investigating the use of LEV for perioperative seizure control in patients with suspected primary brain tumors undergoing neurosurgery (e.g., biopsy or resection) (36). Inclusion criteria included age $>18$ years, neuroradiological imaging suspected primary brain tumor, and planned biopsy or resection neurosurgical procedure. Patients were excluded if in the seven preoperative days any AED other than LEV was used or if there was a known LEV allergy or previous severe side-effects to LEV. Between January
2008 and June 2009, 25 of 30 study patients were enrolled and treated for 4 weeks before and 4 weeks after a neurosurgery procedure with oral and IV LEV. During this time patients had four scheduled visits for monitoring and data collection. Of the 27 patients who underwent surgery, 22 were diagnosed with a primary brain tumor, 3 with meningioma, 2 with brain metastasis, and 1 with abscess. Subjects were started on an oral dose of LEV $500 \mathrm{mg}$ twice daily and titrated to $1,000 \mathrm{mg}$ twice daily after $72 \mathrm{~h}$. The dose was further increased if the patient was at an increased risk of seizures or had seizures in this period. IV LEV was given immediately and for $36 \mathrm{~h}$ postoperatively before being transitioned back to oral LEV. Three patients did not have the planned procedure and two patients were lost to follow up.

In the pre-surgery phase (defined as 3 days- 4 weeks prior to surgery), $100 \%$ of patients were seizure free after the initiation of LEV therapy. In the $48 \mathrm{~h}$ post surgery phase and early follow up phase (defined as $48 \mathrm{~h}-4$ weeks post surgery), the seizure free rates were 88 and $84 \%$, respectively. Three patients failed LEV treatment, even after the dose was titrated up to $3,000 \mathrm{mg} /$ day. There were no serious adverse events reported with LEV treatment. The authors concluded that oral and IV LEV for perioperative seizure control was feasible and safe in patients with tumor-related seizures (36). However, to date only four studies have been able to demonstrate the effectiveness of levetiracetam in the perioperative phase (3235). Zachenhofer et al. (37) retrospectively studied 78 patients with brain tumors who received between 1,000 and 3,000 $\mathrm{mg}$ of LEV perioperatively. After a mean follow up time of 10.5 months, $91 \%$ of the patients were seizure free. This study demonstrated a very low seizure frequency of $2.5 \%$ in the early postoperative period. These studies propose both the feasibility and safety of intravenous levetiracetam in the perioperative treatment, but more long term trials are needed.

\section{INCREASING CHEMOTHERAPY SENSITIVITY}

Levetiracetam's role in increasing chemotherapy sensitivity is a fascinating new field of interest. Some antiepileptic drugs have actually shown that they can inhibit histone deacetylase activity within the tumor. Histone deacetylase inhibitors can modulate temozolomide activity by modulating methylguanine-DNAmethyltransferase (MGMT) expression (37) thereby allowing for increased temozolomide efficacy. Levetiracetam has in fact been shown to increase the transcription of histone deacetylase 1 (HDAC1) which ultimately silences MGMT (38). Levetiracetam has also been shown to have a neuroprotective role via free-radical scavenging activity (39), reducing inflammation and neuronal death (40). All of these attributes may lead to the ability of levetiracetam to prevent radiochemotherapy-caused nerve damage. Levetiracetam may in fact increase temozolomide-induced cytotoxicity in patients with GBM who do express the MGMT protein while also experiencing little adverse side-effects $(41,42)$. This could be a very exciting area of research as GBM tumors are so common.

\section{CONCLUSION}

Brain tumor patients require a multidisciplinary approach involving the use of chemotherapy, radiation, possible surgery, and in many cases antiepileptic drugs. The first-line treatments for 
these patients have numerous drug interactions to be weary of when using them in addition to anticancer drugs. Increased sideeffects have led to renewed interest in antiepileptic drugs that do not induce cytochrome P450 pathways. Levetiracetam, a drug unique in both its mechanism of action and in the way that it does not cause the drug interactions of the first-line drugs, is among the newer antiepileptic drugs. Several studies have demonstrated the effectiveness of levetiracetam in the role of

\section{REFERENCES}

1. Hauser WA, Annegers JF, Kurland LT. Incidence of epilepsy and unprovoked seizures in Rochester, Minnesota: 1935-1984. Epilepsia (1993) 34:453-68. doi:10.1111/j. 1528-1157.1993.tb02586.x

2. Herman ST. Epilepsy after brain insult: targeting epileptogenesis. Neurology (2002) 59(Suppl 5):S21-6. doi:10.1212/WNL.59.9_ suppl_5.S21

3. Glantz MJ, Cole BF, Forsyth PA, Recht LD, Wen PY, Chamberlain MC, et al. Practice parameter: anticonvulsant prophylaxis in patients with newly diagnosed brain tumors. Report of the Quality Standards Subcommittee of the American Academy of Neurology. Neurology (2000) 54:1886-93. doi:10. 1212/WNL.54.10.1886

4. Taphoorn MJ. Neurocognitive sequelae in the treatment of lowgrade gliomas. Semin Oncol (2003) 30:45-8. doi:10.1053/j.seminoncol. 2003.11.023

5. van Breemen RM, Rijsman RM, Taphoorn MJB, Walchenbach R, Zwinkels H, Vecht CJ. Efficacy of anti-epileptic drugs in patients with gliomas and seizures. J Neurol (2009) 256:1519-26. doi:10.1007/ s00415-009-5156-9

6. Moots PL, Maciunas RJ, Eisert DR, Parker RA, Laporte K, Abou-Khalil B. The course of seizure disorders in patients with malignant gliomas. Arch Neurol (1995) 52:717-24. doi:10.1001/archneur.1995. 00540310091021

7. Tsai H, Wei K, Tsai C, Huang Y, Chen $\mathrm{P}$, Chen S, et al. Effect of valproic acid on the outcome of glioblastoma multiforme. $\mathrm{Br} J$ Neurosurg (2012) 26(3):347-54. doi:10.3109/ 02688697.2011 .638996

8. Maschio M, Albani F, Baruzzi A, Zarabla A, Dinapoli L, Pace A, et al. Levetiracetam therapy in patients with brain tumor and epilepsy. $J$ Neurooncol (2006) 80:97-100. doi: 10.1007/s11060-006-9162-9

9. Murry DJ, Cherrick I, Salama V, Berg S, Bernstein M, Kuttesch $\mathrm{N}$, et al. Influence of phenytoin on the disposition of irinotecan: a case report. I Pediatr
Hematol Oncol (2002) 24:130-3. doi:10.1097/00043426-20020200000014

10. Grossman F. A review of anticonvulsants in treating agitated demented elderly patients. Pharmacotherapy (1998) 18:600-6.

11. Maschio M, Dinapoli L. Lecture: profile of risks and benefits of new antiepileptic drugs in brain tumorrelated epilepsy. Neurol Sci (2011) 32(Suppl 2):S259-62. doi:10.1007/ s10072-011-0801-3

12. Van Breemen MS, Vecht CJ. Optimal seizure management in brain tumor patients. Curr Neurol Neurosci Rep (2005) 5:207-13. doi:10. 1007/s11910-005-0048-6

13. Baulac M, Brodie MJ, Elger CE, Krakow K, Stockis A, Meyvisch $\mathrm{P}$, et al. Levetiracetam intravenous infusion as an alternative to oral dosing in patients with partial-onset seizures. Epilepsia (2007) 48:589-92. doi:10.1111/j. 1528-1167.2006.00959.x

14. Maschio M, Dinapoli L, Sperati F, Fabi A, Pace A, Vidri A, et al. Oxcarbazepine monotherapy in patients with brain tumor-related epilepsy: open-label pilot study for assessing the efficacy, tolerability, and impact on quality of life. J Neurooncol (2012) 106:651-6. doi:10.1007/ s11060-011-0689-z

15. Meyer FP, Banditt P, Schubert A, Schoche J. Lamotrigine concentrations in human serum, brain tissue, and tumor tissue. Epilepsia (1999) 40:68-73. doi:10.1111/j.1528-1157. 1999.tb01990.x

16. Swisher C, Doreswamy M, Gingrich K, Vredenburgh J, Kolls B. Phenytoin, levetiracetam, and agement of refractory status epilepticus in patients with brain tumors. Neurocrit Care (2012) 16: 109-13. doi:10.1007/s12028-0119626-4

17. Saria M, Corle C, Hu J, Rudnick J, Phuphanich S, Mrugala $\mathrm{M}$, et al. Retrospective analysis of the tolerability and activity of lacosamide in patients with brain tumors: clinical article. J Neurosurg (2013) 118:1183-7. doi:10. 3171/2013.1.JNS12397 pregabalin in the acute man-

monotherapy as well as adjunct therapy to other antiepileptic drugs. Levetiracetam has also sparked the interest of clinicians for its role in surgical prophylaxis, for refractory status epilepticus, and the ability to increase chemotherapy sensitivity. Some of these attributes have more supporting evidence than others, but levetiracetam does warrant more testing and could very well be a promising drug in the fight to control epilepsy in brain tumor patients.

18. Maschio M, Dinapoli L, Mingoia M, Sperati F, Pace A, Pompili A, et al. Lacosamide as add-on in brain tumor-related epilepsy: preliminary report on efficacy and tolerability. J Neurol (2011) 258:2100-4. doi:10. 1007/s00415-011-6132-8

19. Kaminski RM, Matagne A, Patsalos PN, Klitgaard H. Benefit of combination therapy in epilepsy: a review of the preclinical evidence with levetiracetam. Epilepsia (2009) 50:387-97. doi:10.1111/ j.1528-1167.2008.01713.x

20. Lynch BA, Lambeng N, Nocka K, Kensel-Hammes P, Bajjalieh SM, Matagne A, et al. The synaptic vesicle protein SV2A is the binding site for the antiepileptic drug levetiracetam. Proc Natl Acad Sci U S A (2004) 101:9861-6. doi:10.1073/ pnas.0308208101

21. Yang XF, Weisenfeld A, Rothman SM. Prolonged exposure to levetiracetam reveals a presynaptic effect on neurotransmission. Epilepsia (2007) 48:1861-9. doi:10.1111/j. 1528-1167.2006.01132.x

22. Lyseng-Williamson KA. Levetiracetam: a review of its use in epilepsy. Drugs (2011) 71:489-514.

23. Ruggiero A, Rizzo D, Mastrangelo S, Battaglia D, Attina G, Riccardi R. Interactions between antiepileptic and chemotherapeutic drugs in children with brain tumors: is it time to change treatment? Pediatr Blood Cancer (2010) 54(2):193-8. doi:10.1002/pbc.22276

24. Wagner GL, Wilms EB, Van Donselaar CA, Vecht CHJ. Levetiracetam: preliminary experience in patients with primary brain tumors. Seizure (2003) 12:585-6. doi:10. 1016/S1059-1311(03)00096-7

25. Newton HB, Goldlust SA, Pearl D. Retrospective analysis of the efficacy and tolerability of levetiracetam in brain tumor patients. I Neurooncol (2006) 78:99-102. doi:10.1007/ s11060-005-9070-4

26. Siddiqui F, Wen P, Dworetzky B, Cabello D, Bromfield EB. Use of levetiracetam in patients with brain tumors. Epilepsia (2002) 43(Suppl 7):297.

27. Rosati A, Buttolo L, Stefini R, Todeschini A, Cenzato M,
Padovani A. Efficacy and safety of levetiracetam in patients with glioma: a clinical prospective study. Arch Neurol (2010) 67: 343-6. doi:10.1001/archneurol. 2009.335

28. Dinappoli L, Maschio M, Jandolo B, Fabi A, Pace A, Sperati F, et al. Quality of life and seizure control in patients with brain tumorrelated epilepsy treated with levetiracetam monotherapy: preliminary data of an open-label study. Neurol Sci (2009) 30:353-9. doi:10. 1007/s10072-009-0087-x

29. Pace A, Bove L, Innocenti P, Pietrangeli A, Carapella CM, Oppido P, et al. Epilepsy and gliomas: incidence and treatment in 119 patients. J Exp Clin Cancer Res (1998) 17:479-82.

30. Liigant A, Haldre S, Oun A, Linnamagi U, Saar A, Asser T, et al. Seizure disorders in patients with brain tumors. Eur Neurol (2001) 45:46-51. doi:10.1159/000052089

31. Stevens GHJ, Vogelbaum MA, Suh JH, Peereboom DM, Barnett GH. Levetiracetam use in brain tumor patients. Neurology (2005) 64: A48.

32. Maschio M, Dinapoli L, Sperati F, Pace A, Fabi A, Vidiri A, et al. Levetiracetam monotherapy in patients with brain tumor related epilepsy: seizure control, safety and quality of life. I Neurooncol (2011) 104:205-14. doi:10.1007/ s11060-010-0460-x

33. Hildebrand J, Lecaille C, Perennes J, Delattre JY. Epileptic seizures during follow-up of patients treated for primary brain tumors. Neurology (2005) 65:212-5. doi:10.1212/ 01.wnl.0000168903.09277.8f

34. DeLorenzo RJ, Hauser WA, Towne AR, Boggs JG, Pellock JM, Penberthy $\mathrm{L}$, et al. A prospective populationbased epidemiologic study of status epilepticus in Richmond, Virginia. Neurology (1996) 46: 1029-35. doi:10.1212/WNL.46.4. 1029

35. Claassen J, Hirsch LJ, Mayer SA. Treatment of status epilepticus: a survey of neurologists. J Neurol Sci (2003) 211:37-41. doi:10.1016/ S0022-510X(03)00036-4 
36. Bähr O, Hermisson M, Rona S, Rieger J, Nussbaum S, Körtvelyessy $\mathrm{P}$, et al. Intravenous and oral levetiracetam in patients with a suspected primary brain tumor and symptomatic seizures undergoing neurosurgery: the HELLO trial. Acta Neurochir (Wien) (2012) 154:229-35. doi:10.1007/s00701011-1144-9

37. Zachenhofer I, Donat M, Oberndorfer S, Roessler K. Perioperative levetiracetam for prevention of seizures in supratentorial brain tumor surgery. $J$ Neurooncol (2011) 101:101-6. doi: 10.1007/s11060-010-0235-4

38. Milligan TA, Hurwitz S, Bromfield EB. Efficacy and tolerability of levetiracetam versus phenytoin after supratentorial neurosurgery. Neurology (2008) 71:665-9. doi:10. 1212/01.wnl.0000324624.52935.46

39. Strahl BD, Allis CD. The language of covalent histone modifications. Nature (2000) 403:41-5. doi:10. 1038/47412

40. Bobustuc GC, Baker CH, Limaye A, Jenkins WD, Pearl G, Avgeropoulos NG, et al. Levetiracetam enhances p-53 mediated MGMT inhibition and sensitizes glioblastoma cells to temozolomide. Neuro Oncol (2010) 12:917-27. doi:10.1093/neuonc/noq044

41. Ueda Y, Doi T, Takaki M, Nagatomo K, Nakajima A, Willmore LJ. Levetiracetam enhances endogenous antioxidant in the hippocampus of rats: in vivo evaluation by brain microdialysis combined with ESR spectroscopy. Brain Res (2009) 1266:1-7. doi:10.1016/j.brainres.2009.02.040
42. Cardile V, Pavone A, Gulino R, Renis M, Scifo C, Perciavalle V. Expression of brain-derived neurotrophic factor (BDNF) and inducible nitric oxide synthase in rat astrocyte cultures treated with levetiracetam. Brain Res (2003) 976:227-33. doi:10.1016/S0006-8993(03) 02720-3

Conflict of Interest Statement: The authors declare that the research was conducted in the absence of any commercial or financial relationships that could be construed as a potential conflict of interest.

Received: 07 May 2013; accepted: 20 September 2013; published online: 07 October 2013.
Citation: Fonkem E, Bricker P, Mungall $D$, Aceves J, Ebwe E, Tang $W$ and Kirmani $B$ (2013) The role of levetiracetam in treatment of seizures in brain tumor patients. Front. Neurol. 4:153. doi: 10.3389/fneur.2013.00153

This article was submitted to Epilepsy, a section of the journal Frontiers in Neurology.

Copyright (c) 2013 Fonkem, Bricker, Mungall, Aceves, Ebwe, Tang and Kirmani. This is an open-access article distributed under the terms of the Creative Commons Attribution License (CC BY). The use, distribution or reproduction in other forums is permitted, provided the original author(s) or licensor are credited and that the original publication in this journal is cited, in accordance with accepted academic practice. No use, distribution or reproduction is permitted which does not comply with these terms. 